\title{
Elevated Interleukin-12 in Progressive Multiple Sclerosis Correlates with Disease Activity and Is Normalized by Pulse Cyclophosphamide Therapy
}

\author{
Manuel Comabella, Konstantine Balashov, Shohreh Issazadeh, Derek Smith, Howard L. Weiner, and Samia J. Khoury \\ Center for Neurologic Diseases, Brigham and Women's Hospital, Boston, Massachusetts 02115
}

\begin{abstract}
Multiple sclerosis is postulated to be a Th1-type cell-mediated autoimmune disease. We investigated cytokine profiles in patients with progressive multiple sclerosis by using intracytoplasmic staining. We found increased IL-12 production by monocytes and increased IFN- $\gamma$ production by $\mathrm{T}$ cells in untreated patients as compared with controls. In patients treated with methotrexate, methylprednisolone, or cyclophosphamide/methylprednisolone (CY/MP), only CY/MP treatment normalized the elevated IL-12 production. Furthermore, CY/MP-treated patients had decreased IFN- $\gamma$ and increased IL-4, IL-5, and TGF- $\beta$ expression. Patients followed prospectively before and after starting CY/MP treatment showed a gradual decrease in IL-12 and IFN- $\gamma$ production and an increase in IL-4 and IL-5. In vitro, addition of 4-hydroperoxycyclophosphamide, a metabolite of cyclophosphamide decreased IL-12 production in mononuclear cell cultures. When patients were classified as having active or stable disease, IL-12 production correlated with disease activity. In summary, our results demonstrate a Th1-type cytokine bias in peripheral blood mononuclear cells of untreated progressive MS patients that is reversed by CY/MP treatment and is associated with Th2 and TGF- $\beta$ (Th3) type responses. These findings provide a basis for immune monitoring of patients with MS and suggest that treatments that downregulate IL-12 may prove to be beneficial in progressive MS. (J. Clin Invest. 1998. 102:671-678.) Key words: intracellular immunofluorescence $\cdot$ cyclophosphamide $\bullet$ interleukin-12 $\bullet$ Th2 $\bullet$ Th3 $\bullet$ multiple sclerosis
\end{abstract}

\section{Introduction}

Multiple sclerosis (MS $)^{1}$ is a chronic inflammatory disease of the central nervous system characterized by perivascular $\mathrm{T}$ cell and macrophage infiltrates leading to demyelination. Al-

Address correspondence to Samia J. Khoury, M.D., Center for Neurologic Diseases, Brigham and Women's Hospital, Harvard Medical School, 77 Louis Pasteur Ave., Boston, MA 02115. Phone: 617-5255370; FAX: 617-525-5252; E-mail: khoury@cnd.bwh.harvard.edu

Received for publication 16 February 1998 and accepted in revised form 17 June 1998.

1. Abbreviations used in this paper: 4HC, 4-hydroperoxycyclophosphamide; CY/MP, cyclophosphamide/methylprednisolone; EAE, experimental autoimmune encephalomyelitis; EDSS, Expanded Disability Status Scale; MS, multiple sclerosis; NK, natural killer; PE, phycoerythrin; rhIFN- $\gamma$, recombinant human IFN- $\gamma$.

J. Clin. Invest.

(C) The American Society for Clinical Investigation, Inc. 0021-9738/98/08/0671/08 \$2.00

Volume 102, Number 4, August 1998, 671-678

http://www.jci.org though the etiology of MS is unknown, it is considered to be a T cell-mediated autoimmune disease (1). In the mouse system, two distinct $\mathrm{T}$ cell subsets have been defined. Th1 cells secrete IL-2 and IFN- $\gamma$ and mediate delayed-type hypersensitivity, whereas Th2 cells secrete predominantly IL-4, IL-5, and IL-10 and mediate humoral immunity (2). Cells that secrete predominantly TGF- $\beta$ have been termed Th3 cells $(3,4)$. In the experimental autoimmune encephalomyelitis (EAE) mouse model, $\mathrm{T}$ cells producing Th1 cytokines can transfer disease $(5,6)$, while spontaneous recovery from EAE correlates with a switch to TGF- $\beta$ and Th2 cytokines (7-10). IL-12 is a heterodimeric cytokine produced mostly by phagocytic cells and induces cytokine production, primarily of IFN- $\gamma$, from T cells. Several studies in humans $(11,12)$ and in the mouse $(13,14)$ have assigned a role to IL-12 as the promoter of Th1 cell generation, acting in antagonism with IL-4, the major promoter of Th2 responses. Administration of IL-12 to mice after transfer of encephalitogenic cells resulted in increased severity and duration of EAE; treatment with anti-IL-12 antibodies substantially reduced the incidence and severity of adoptively transferred EAE (15). Elevated serum levels of IL-12 as well as increase in T cell receptor-mediated IL-12 secretion have been reported in the chronic progressive form of MS $(16,17)$. The results of these studies suggest that IL-12 plays an important role in the pathogenesis of EAE and MS and that immune strategies aimed at downregulating IL-12 may be efficacious therapies.

Cyclophosphamide is a cytotoxic drug that interferes with DNA synthesis and has its major pharmacological action on dividing cells. Cyclophosphamide was one of the first drugs shown to reverse the course of EAE even when given after symptoms appeared $(18,19)$ and provided a rationale for treating patients with MS. Early studies on the immunologic effects of cyclophosphamide demonstrated a decrease in the absolute number of T cells (20), a reduction in circulating B cells (21), a decrease in the synthesis of IgG within the blood-brain barrier (22), and decreased spontaneous proliferation of lymphocytes (23). We have investigated the use of pulse cyclophosphamide to treat progressive MS and found a positive clinical effect in younger patients with actively progressive disease of short duration $(24,25)$, although not all studies of cyclophosphamide therapy found a positive effect (26). Moreover, we reported an increase in the anti-CD3-induced IL-4 secretion by T cells in MS patients treated with pulse cyclophosphamide methylprednisolone (CY/MP; 27) and have recently found that CY/MP induces myelin antigen specific IL-4-secreting $\mathrm{T}$ cells in MS patients (28). Other immunosuppressive drugs such as methotrexate, cladribine, and monthly intravenous methylprednisolone have also been used to treat progressive MS patients, and the mechanism by which these agents may affect disease process in MS is also unknown.

Several techniques have been used to analyze cytokine synthesis ex vivo at the single-cell level: ELISPOT, in situ hybridization, limiting dilution, and single-cell PCR (29). However, 
all of these techniques have significant drawbacks, requiring either high technical proficiency or laborious manual scoring of data. Cytokine production measured by intracytoplasmic staining and flow cytometry has the advantage of rapidly determining the cytokine production of a large number of individual cells. In addition, flow cytometry allows the identification of the phenotype and quantification of the frequency of cytokine producing cells as defined by membrane antigens and intracellular cytokines. We used this new approach to analyze a panel of cytokines in the peripheral blood of progressive MS patients with or without immunosuppressive therapies. We found that IL-12 production was linked to disease activity in patients with progressive disease, and that a decrease in IL-12 production with an increase in Th2 cytokines and TGF- $\beta$ was associated with $\mathrm{CY} / \mathrm{MP}$ treatment.

\section{Methods}

Patients and treatment regimens. MS patients were followed at the outpatient MS clinic at the Brigham and Women's Hospital. Neurologic examination including physician assessment of disease activity and scoring on the Expanded Disability Status Scale (EDSS) was performed at every visit. There were 18 untreated progressive MS patients who had never received immunosuppressive therapy and who had not received steroid treatment in the 6 mo before blood drawing $($ female $/$ male $=12: 6$; average age $=50.2 \pm 2.5$; average EDSS $=$ $5.6 \pm 0.3)$. Patients on pulse cyclophosphamide received $600-1,200$ $\mathrm{mg} / \mathrm{m}^{2}$ intravenous cyclophosphamide every 4-8 wk together with 1 gm of intravenous methylprednisolone (CY/MP). Cyclophosphamide dose was adjusted to produce a white blood cell nadir of 2,000 cells/ $\mathrm{cm}^{3} 10-14 \mathrm{~d}$ after therapy. 21 patients treated with pulse cyclophosphamide were studied $(\mathrm{F} / \mathrm{M}=18: 3$; average age $=42.7 \pm 2.0$; average EDSS $=5.1 \pm 0.3) .11$ progressive MS patients were receiving monthly infusions of intravenous methylprednisolone only $(\mathrm{F} / \mathrm{M}=$ $5: 6$; average age $=49.9 \pm 3.8$; average EDSS $=5.7 \pm 0.3) .11$ progressive MS patients were receiving methotrexate $(\mathrm{F} / \mathrm{M}=8: 3$; average age $=50.7 \pm 3.8$; average EDSS $=5.7 \pm 0.3)$. The control group consisted of 14 healthy individuals $(\mathrm{F} / \mathrm{M}=6: 8$, average age $=44.6 \pm 3.6)$. Disease activity was assessed by the examining physician at the time of blood drawing, and each patient rated as stable or active based on clinical history and neurologic examination. A longitudinal study was performed in three progressive MS patients with active disease before starting treatment with pulse cyclophosphamide and monthly for 3 mo after starting treatment of monthly CY/MP pulses. Three healthy donors were also followed as controls for the same time. Peripheral blood was collected immediately before cyclophosphamide infusion in patients followed prospectively.

Antibodies and reagents. FITC-conjugated mouse anti-human IgG2a CD14 and Cy-Chrome mouse anti-human IgG2a CD3 were obtained from PharMingen (San Diego, CA). FITC and Cy-Chrome mouse IgG2a (PharMingen) were used as isotype controls. The following $\mathrm{mAb}$ against intracytoplasmic cytokines (all phycoerythrin [PE]-conjugated) were obtained from PharMingen: mouse anti-human IgG1 p40/p70 IL-12, mouse anti-human IgG1 IFN- $\gamma$, mouse anti-human IgG1 TNF- $\alpha$, mouse anti-human IgG1 IL-4, rat anti-human IgG2a IL-10, and rat anti-human IgG2a IL-5. PE-conjugated mouse IgG1 and rat IgG2a were used as isotype controls. Unlabeled mouse anti-human IL-12 was used as specificity control. The following FITC-conjugated antibodies were also obtained from PharMingen: mouse anti-human IgG1 CD40, mouse anti-human IgG1 CD86, and mouse anti-human IgG2a class II MHC. Mouse IgG1 and mouse IgG2a, both FITC-conjugated, were used as isotype controls. Mouse anti-human IgG1 CD80 was from Immunotech (Westbrook, ME). Purified mouse antihuman IgG1 TGF- $\beta 1 \mathrm{mAb}$ was obtained from R\&D Systems (Gaithersburg, MD). Purified mouse anti-human IgG1 (PharMingen) was used as isotype control. PE-conjugated goat anti-mouse IgG1 (Caltag Labs, South San Francisco, CA) was used as a secondary reagent.

Phorbol 12-myristate 13-acetate (PMA), ionomycin, LPS, saponin, and monensin were obtained from Sigma Chemical Co. (St. Louis, MO); PHA was from Murex Diagnostics Limited (Dartford, England); recombinant human IFN- $\gamma($ rhIFN- $\gamma$ ) was from PharMingen. 4-Hydroperoxycyclophosphamide (4HC) was kindly provided by Michael Colvin (Johns Hopkins Oncology Center, Baltimore, MD).

Cell separation and culture conditions. PBMC were isolated by Ficoll-Hypaque density gradient centrifugation and resuspended $(2 \times$ $10^{6} / \mathrm{ml}$ ) in complete culture media consisting of RPMI 1,640 medium supplemented with $10 \%$ fetal bovine serum, $4 \mathrm{mM}$ L-glutamine, 25 $\mathrm{mM}$ Hepes buffer, 50 units $/ \mathrm{ml}$ penicillin, and $50 \mathrm{mg} / \mathrm{ml}$ streptomycin (all from BioWhittaker, Walkersville, MD). Optimal conditions for mononuclear cell stimulation were determined by kinetic studies using single stimulating agents or combination of agents. The time of maximal cytokine production was used for the studies reported below. Maximal stimulation of monocytes for IL-12 production was obtained with sequential stimulation with IFN- $\gamma$ and LPS as follows: PBMC were placed in polypropylene culture tubes (Fisher Scientific Co., Pittsburgh, PA) in a volume of $1 \mathrm{ml}$ and stimulated with rhIFN- $\gamma$ $(10 \mathrm{ng} / \mathrm{ml})$ for $2 \mathrm{~h}$ and LPS $(100 \mathrm{ng} / \mathrm{ml})$ for $22 \mathrm{~h}$. To determine IL-10, TNF- $\alpha$, and TGF- $\beta$ secretion, cells were cultured either in medium alone or with LPS $(100 \mathrm{ng} / \mathrm{ml})$ for $6 \mathrm{~h}$. Maximal T cell cytokine production was achieved with a combination of PHA, PMA, and ionomycin as follows: to determine IL-4, IL-5, IL-10, IFN- $\gamma$, TNF- $\alpha$, and TGF- $\beta$ by T cells, PBMC were placed in 6-well plates (Costar Corp., Cambridge, MA) in a volume of $6 \mathrm{ml}$ and cultured in medium alone or with a combination of PMA $(100 \mathrm{ng} / \mathrm{ml})$, ionomycin $(250 \mathrm{ng} / \mathrm{ml})$, and PHA $(1 \mu \mathrm{g} / \mathrm{ml})$ for $6 \mathrm{~h}$. In all cases, monensin $(3 \mu \mathrm{M})$ was added to the PBMC cultures from the beginning to inhibit cytokine secretion.

Intracytoplasmic staining. Cultured cells were washed in staining buffer (DPBS without $\mathrm{Mg}^{2+}$ or $\mathrm{Ca}^{2+}, 0.1 \%$ sodium azide, $1 \%$ heatinactivated FCS) and resuspended in staining buffer containing $20 \%$ of human antibody serum (Sigma) to block Fc receptors. Antibodies to cell surface markers were added for $30 \mathrm{~min}$ at the end of the stimulation culture, i.e., at $24 \mathrm{~h}$ for IL-12 cultures and at $6 \mathrm{~h}$ for other cytokine cultures. The cells were washed twice in staining buffer then fixed with $4 \%$ paraformaldehyde and left for $20 \mathrm{~min}$ or overnight in the dark. Cells were washed twice, resuspended in permeabilization buffer (DPBS without $\mathrm{Mg}^{2+}$ or $\mathrm{Ca}^{2+}, 0.1 \%$ sodium azide, $1 \%$ heatinactivated FCS, $0.1 \%$ saponin) and incubated with antibodies against cytokines or isotype controls in a concentration ranging from 0.25 to 1 $\mu \mathrm{g}$ per $10^{6}$ cells for $30 \mathrm{~min}$. The cells were washed in permeabilization buffer then twice in staining buffer. For TGF- $\beta$ staining, incubation with anti-TGF- $\beta$ was followed by incubation with goat anti-mouse antibody (1/25 dilution) for $10 \mathrm{~min}$. Purified mouse IgG1 plus PElabeled goat anti-mouse served as negative control. All staining steps were carried out at $4^{\circ} \mathrm{C}$ in the dark. Cytokine blocking experiments were performed by the addition of an excess of unlabeled anti-cytokine $\mathrm{mAb}$ for $20 \mathrm{~min}$ before the addition of the labeled anti-cytokine $\mathrm{mAb}$ (Fig. 1).

In vitro experiments using 4HC. PBMC $\left(2 \times 10^{6} / \mathrm{ml}\right)$ were cultured in polypropylene tubes for $1 \mathrm{~h}$ with or without $4 \mathrm{HC}$ at $10^{-9}$, $10^{-7}$, and $10^{-5} \mathrm{M}$. Cells were washed three times in complete media and stimulated with either LPS for $6 \mathrm{~h}$ or rhIFN- $\gamma$ plus LPS for $24 \mathrm{~h}$. Intracytoplasmic staining of TNF- $\alpha$ and IL-10 or IL-12 was performed as previously described. In additional experiments, monocytes were purified based on CD14 expression and pretreated with $4 \mathrm{HC}$ for $1 \mathrm{~h}$ then stimulated with rhIFN- $\gamma$ plus LPS for $24 \mathrm{~h}$ and stained for IL-12. Isolation of CD14 ${ }^{+}$monocytes was performed by incubation of cells with mouse anti-human CD14 microbeads (Miltenyi Biotec Inc., Bergisch Gladbach, Germany). Positive selection of $\mathrm{CD} 14^{+}$cells was performed by magnetic cell separation using the MiniMACS $^{\mathrm{TM}}$ system (Miltenyi Biotec Inc.). The purity of CD14 monocytes was greater than $95 \%$ as assessed by flow cytometry.

Surface staining for CD40, CD80, CD86, and Class II MHC was performed on purified monocytes after incubation with $4 \mathrm{HC}$ as 
A

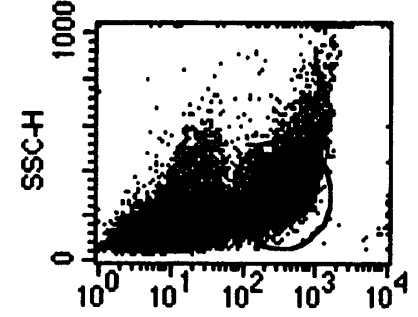

B

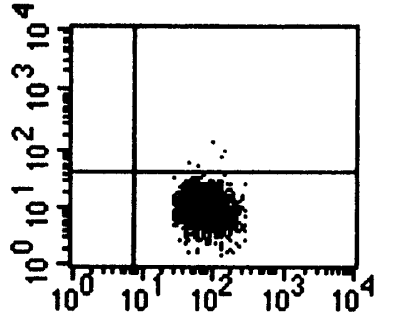

C

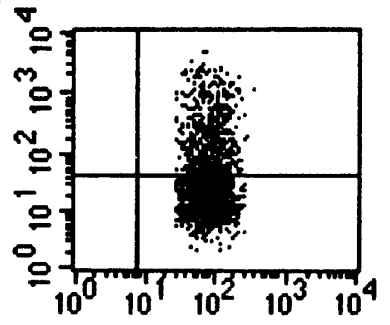

D.

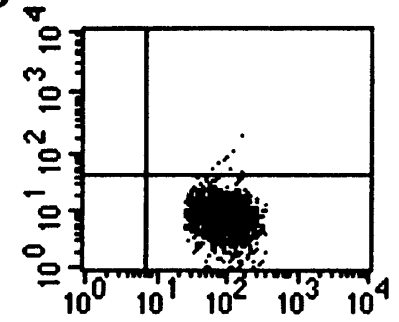

Figure 1. Intracellular staining of IL-12 in monocytes. $(A)$ Monocyte gate on CD14 ${ }^{+}$cells. $(B)$ Negative control using mouse IgG1 antibodies. (C) Specific IL-12 staining showing the percentage of CD14 ${ }^{+}$cells that express IL-12. (D) Specificity control: IL-12 staining was blocked by preincubation of fixed and permeabilized cells with excess unlabeled anti-IL-12 antibody for 20 min before the addition of labeled anti-IL-12 antibody. $S S C$, side scatter.

above. In brief, after $18 \mathrm{~h}$ of stimulation (time for maximal expression of costimulatory molecules), the cells were washed in staining buffer, incubated with antibodies for $30 \mathrm{~min}$, and then washed twice in staining buffer and fixed with $1 \%$ paraformaldehyde.

Flow cytometry. Cells were analyzed using a FACSort flow cytometer (Becton Dickinson Immunocytometry Systems, San Jose, CA) equipped with CellQuest software. Cytokine expression by $\mathrm{T}$ cells and monocytes was determined based on CD3 and CD14 staining. 20,000 events (for T cells) and 40,000 events (for monocytes) were acquired. The percentage of cytokine positive cells was calculated by subtracting the isotype control antibody signal from the specific anti-cytokine antibody signal.

Statistical analysis. The Kruskal-Wallis test was used to analyze differences in cytokine production among groups. If significant differences $(P<0.05)$ were found, a two-tailed unpaired Mann-Whitney's test was then used to test for significant differences between two groups. Results are presented as mean \pm SEM values.

\section{Results}

Increased IL-12 expression in patients with progressive MS. We investigated the production of IL-12 by monocytes of untreated progressive MS patients. Under unstimulated conditions very few cells $(<1 \%)$ produce IL-12 in MS patients or normal individuals. Thus, intracellular staining for IL-12 was performed in 61 patients and 14 controls after rhIFN- $\gamma$ plus LPS stimulation. As seen in Fig. 2, patients with progressive MS on no treatment $(n=18)$ had a significantly $(P=0.005)$ higher percentage of IL-12 secreting monocytes (mean $26.3 \pm 2.7 \%$ ) than healthy individuals $(n=14$, mean $14.9 \pm 2.1 \%)$. Patients with progressive MS treated with MP or methotrexate had levels similar to the untreated patients, whereas patients treated with CY/MP had decreased percentage of monocytes expressing IL-12 to the level seen in healthy individuals (mean $13.0 \pm 1.8 \%$ ). We investigated whether other PBMCs may produce IL-12; natural killer (NK) cells produced no detectable IL-12 before or after stimulation, and less than $1 \%$ of B cells produced IL-12 after LPS stimulation in normal individuals and MS patients.

Measurement of TNF- $\alpha$ and IL-10 production by monocytes under stimulated and unstimulated conditions (Table I) showed no significant differences among patients (treated or untreated and controls).

Increased IFN- $\gamma$ production by $T$ cells from untreated progressive MS patients and normalization of Th1 and Th2 cytokine balance in treated patients. PBMCs from patients and healthy controls were stimulated in vitro for $6 \mathrm{~h}$ with a combination of
PMA (100 ng/ml), ionomycin $(250 \mathrm{ng} / \mathrm{ml})$, and PHA $(1 \mu \mathrm{g} / \mathrm{ml})$. Intracellular staining for IL-4, IL-5, IL-10, IFN- $\gamma$, and TNF- $\alpha$ was measured in T cells (Table II).

Under unstimulated conditions, T cells expressed IL-4 and IL-5 while IFN- $\gamma$ and TNF- $\alpha$ were not detectable. In patients treated with MP, the percentage of IL-4-expressing cells was significantly higher than in untreated progressive MS patients $(P=0.01)$. In patients treated with CY/MP, there were significantly higher percentages of IL-4- and IL-5-producing cells

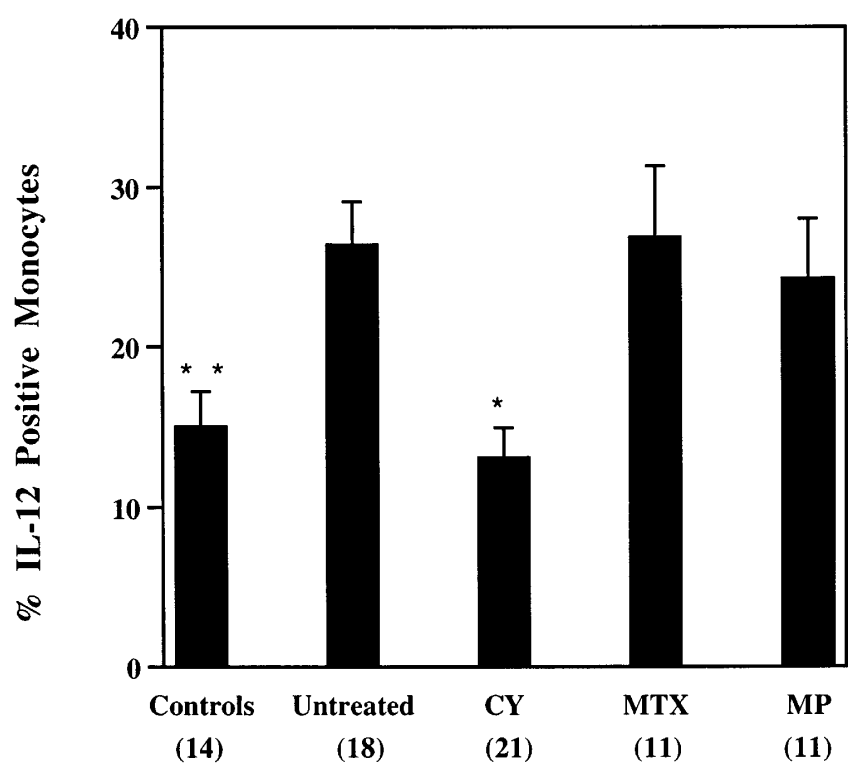

Figure 2. LPS-stimulated IL-12 expression in progressive MS patients and healthy controls. $2 \times 10^{6} \mathrm{PBMC}$ were primed with rhIFN- $\gamma$ for $2 \mathrm{~h}$ and then activated with LPS for $22 \mathrm{~h}$. Monensin was added in the last $22 \mathrm{~h}$ to suppress the secretion of cytokines. Cells were incubated with anti-CD14 antibody and fixed and permeabilized before staining with anti-IL-12 antibody. Data represent the percentage of IL-12+ CD14 cells for each patient. $C Y$, cyclophosphamide/methylprednisolone; $M T X$, methotrexate; $M P$, methylprednisolone. IL-12 expression was significantly decreased in the cyclophosphamide group $(* P=0.0005$ vs. untreated progressive MS; $P=0.007$ vs. MTX; $P=0.01$ vs. MP) and similar to that of the control group $(* * P=0.005$ vs. untreated progressive MS; $P=0.02$ vs. MTX; $P=0.03$ vs. MP). Error bars indicate SEM for each group (controls, $14.9 \pm 2.1$; untreated progressive MS, 26.3 \pm 2.7 ; MTX 26.9 \pm 4.4 ; MP 24.27 \pm 3.7 ; CY $13.06 \pm 1.8)$. 
Table I. Percentage of TNF- $\alpha$ and IL-10 Positive Monocytes

\begin{tabular}{lrrrrr}
\hline & \multicolumn{2}{c}{ Unstimulated } & & \multicolumn{2}{c}{ LPS-stimulated } \\
\cline { 2 - 3 } & \multicolumn{1}{c}{ TNF- $\alpha$} & IL-10 & & TNF- $\alpha$ & IL-10 \\
\hline Control $(n=8)$ & $7.7 \pm 5.1$ & $1.7 \pm 1.4$ & & $78.9 \pm 3.1$ & $17.8 \pm 2.9$ \\
Untreated $(n=8)$ & $17.3 \pm 9.7$ & $3.0 \pm 1.6$ & & $74.3 \pm 5.5$ & $18.9 \pm 3.9$ \\
MTX $(n=7)$ & $8.3 \pm 3.9$ & $2.0 \pm 1.2$ & & $80.8 \pm 3.8$ & $20.4 \pm 2.4$ \\
MP $(n=8)$ & $14.0 \pm 4.6$ & $3.0 \pm 1.2$ & & $78.1 \pm 2.0$ & $15.7 \pm 4.0$ \\
CY $(n=8)$ & $9.3 \pm 4.6$ & $4.9 \pm 2.0$ & & $81.0 \pm 3.3$ & $18.3 \pm 2.7$
\end{tabular}

Results are expressed as mean \pm SEM. $M T X$, methotrexate; $M P$, methylprednisolone; $C Y$, cyclophosphamide.

compared with the other groups (Table II). IL-10 production by $\mathrm{T}$ cells did not differentiate among groups.

After mitogenic stimulation, IFN- $\gamma$ production by $\mathrm{T}$ cells was increased in untreated progressive MS patients $(P=0.03$ compared with normal controls). In contrast, both CY/MP and methylprednisolone treatments were associated with a significant reduction $(P=0.004$ and 0.04 compared with untreated patients, respectively) in IFN- $\gamma$ expression (Table II). No differences in IL-10, IL-4, IL-5, and TNF- $\alpha$ expression was observed among groups after mitogenic stimulation.

$T G F-\beta$ expression by $T$ cells and monocytes is increased in treated patients. TGF- $\beta$ expression by $\mathrm{T}$ cells and monocytes was determined in PBMC after $6 \mathrm{~h}$ incubation with and without the appropriate stimulation, as described above. The percentage of T cells expressing TGF- $\beta$ in controls and untreated progressive MS was $<1 \%$. Immunosuppressive treatment was associated with an increase in TGF- $\beta$ expression, reaching statistical significance in methotrexate $(1.2 \pm 0.3, P=0.001$ vs. controls) and CY/MP-treated patients (2.0 $\pm 1.2, P=0.0003$ vs. controls; $P=0.02$ vs. untreated progressive MS; Fig. 3). Baseline TGF- $\beta$ expression by monocytes was $<2 \%$ in all groups except the CY/MP treatment group where the percentage of monocytes secreting TGF- $\beta$ was significantly higher (4.5 \pm 1.5$)$ compared with controls $(P=0.02)$ and methotrexate groups $(P=0.02$; Fig. 3$)$. No differences in TGF- $\beta$ expression were observed after mitogenic stimulation.

Prospective study of patients before and after cyclophosphamide treatment. To establish that the observed changes in cytokine expression were linked to treatment we followed three active progressive MS patients before starting treatment with

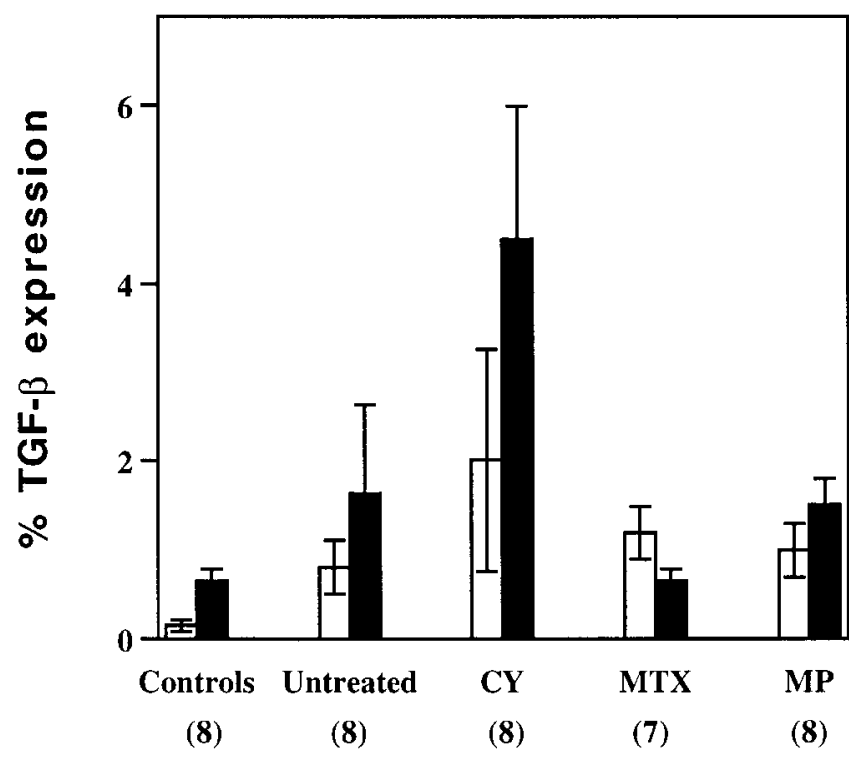

Figure 3. TGF- $\beta$ production by T cells and monocytes is increased by cyclophosphamide treatment. PBMC were cultured for $6 \mathrm{~h}$ without mitogenic stimulation and stained for TGF- $\beta$. TGF- $\beta$ expression was compared among progressive MS groups ( $C Y$, cyclophosphamide/ methylprednisolone; $M T X$, methotrexate; $M P$, methylprednisolone) and healthy controls ( $\mathrm{x}$-axis). TGF- $\beta$ production by unstimulated $\mathrm{CD}^{+}$cells in culture (clear bars) and unstimulated $\mathrm{CD} 14^{+}$cells in culture (black bars) is presented as percentage of positive cells (y-axis). For T cell TGF- $\beta$, cyclophosphamide vs. controls $P=0.0003$ and vs. untreated patients $P=0.02$, MTX vs. controls $P=0.001$. For TGF- $\beta$ in monocytes, cyclophosphamide vs. controls or MTX $P=0.02$.

CY/MP and for 3 mo after treatment. Three healthy controls were also followed for the same time. The time course of cytokine changes before and after treatment is shown in Fig. 4. IL-12 production by monocytes and IFN- $\gamma$ expression by T cells in MS patients were elevated compared with control before CY/MP treatment and decreased after therapy started. No significant changes in IL-12 or IFN- $\gamma$ production were seen in the healthy controls over the same period. On the other hand, IL-4 and IL-5 expression in MS patients was comparable with control before CY/MP treatment and increased during the follow-up period. These cytokine changes were noted after the first month of treatment. IL-4 and IL-5 expression remained stable in the controls (Fig. 4).

Table II. Percentage of Cytokine Positive CD3 Cells

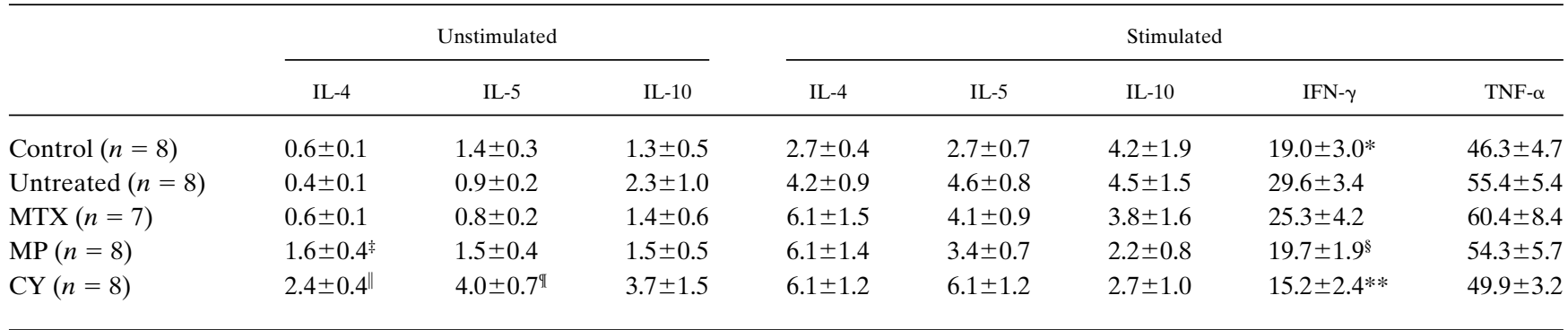

$* P=0.03$ vs. untreated MS. ${ }^{\ddagger} P=0.01$ vs. untreated MS. ${ }^{\S} P=0.04$ vs. untreated MS. $\|^{\|} P=0.0002$ vs. untreated MS, $P=0.0003$ vs. MTX, $P=0.001$ vs. controls. ${ }^{\mathbb{I}} P=0.006$ vs. untreated MS, $P=0.004$ vs. MTX, $P=0.009$ vs. controls or MP. $* * P=0.004$ vs. untreated MS, $P=0.04$ vs. MTX. Results are expressed as mean \pm SEM. $M T X$, methotrexate; $M P$, methylprednisolone; $C Y$, cyclophosphamide. 

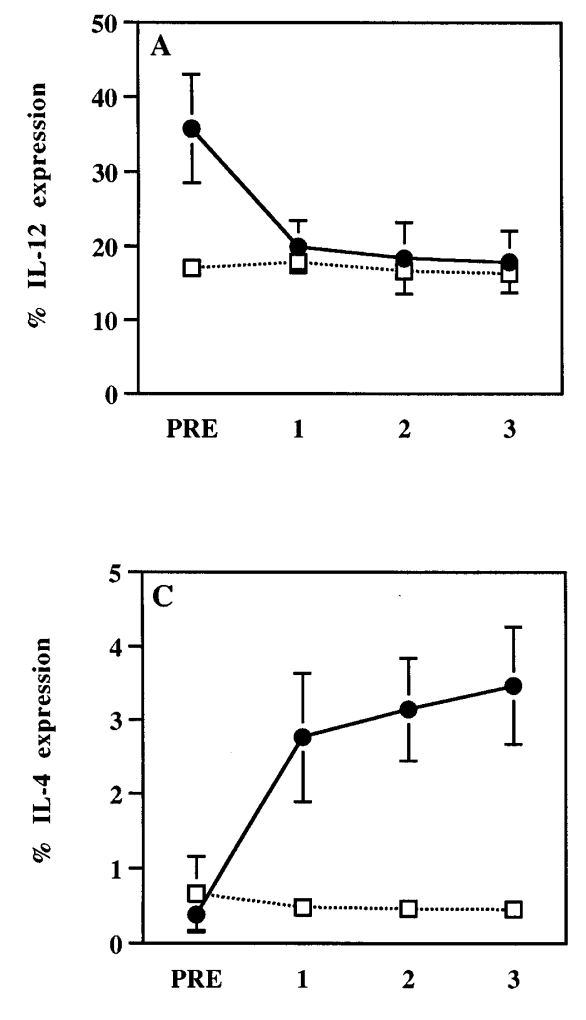

Month After Treatment
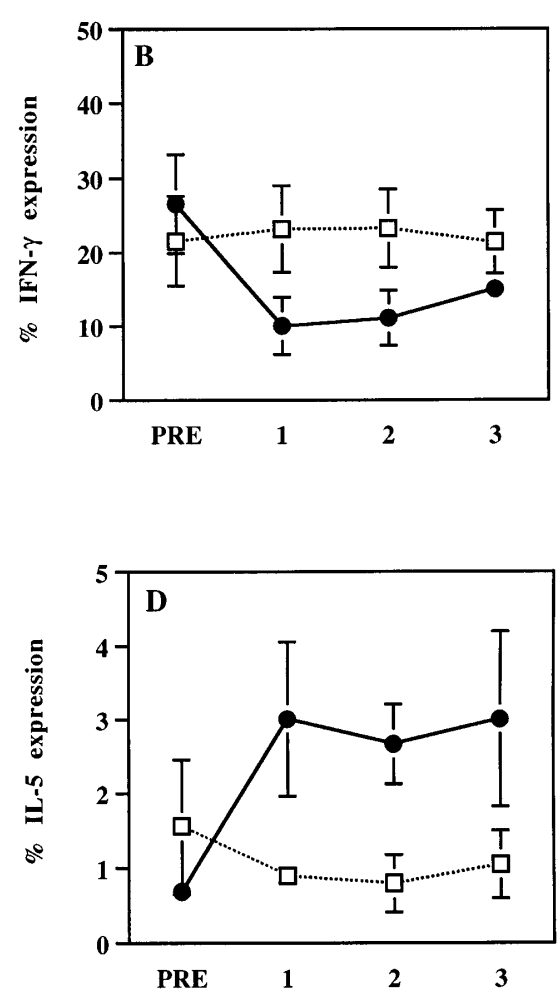

Figure 4. Prospective study of cytokine production during cyclophosphamide treatment. Cytokine production by monocytes and $\mathrm{T}$ cells were determined before starting cyclophosphamide $(P R E)$ and monthly for the first 3 mo of treatment (x-axis). Filled symbols represent data from MS patients, and open circles are from healthy individuals studied at the same time. IL-12 production was determined in $\mathrm{CD} 14^{+}$cells after stimulation with IFN- $\gamma$ and LPS. IFN- $\gamma$ production by $\mathrm{CD}^{+}$cells was determined after PMA, PHA, and ionomycin stimulation. IL-4 and IL-5 production are from unstimulated, cultured $\mathrm{CD}^{+}$cells. The results are the mean \pm SEM of three MS patients and three healthy controls.
In vitro pretreatment of PBMC with $4 H C$ decreased IL-12 expression. To investigate the effect of cyclophosphamide on IL-12 production, we studied the effects of $4 \mathrm{HC}-$ one of the known cyclophosphamide metabolites-on monocytes in vitro. PBMCs were cultured for $1 \mathrm{~h}$ with different concentrations of the metabolite before stimulation with rhIFN- $\gamma$ and LPS for 24 h. As shown in Fig. 5, IL-12 expression decreased by $\sim 50 \%$ with the addition of $4 \mathrm{HC}$. The inhibition was dose dependent and was not due to decreased viability of monocytes, since the number of cells recovered from cultures were similar in the cultures with and without $4 \mathrm{HC}$. No effect of $4 \mathrm{HC}$ on IL-10 or
TNF- $\alpha$ expression by monocytes was seen after LPS stimulation for $6 \mathrm{~h}$.

To determine whether $4 \mathrm{HC}$ acts directly on monocytes, we performed experiments in which monocytes were purified based on CD14 expression, pretreated with $4 \mathrm{HC}$ for $1 \mathrm{~h}$, and then stimulated for $24 \mathrm{~h}$. As shown in Fig. 5 A, IL-12 expression by purified monocytes after rhIFN- $\gamma$ and LPS stimulation was lower compared with IL-12 production following stimulation of PBMC. Nonetheless, the addition of $4 \mathrm{HC}$ to purified monocytes was associated with a small dose-dependent decrease in IL-12 expression.
A

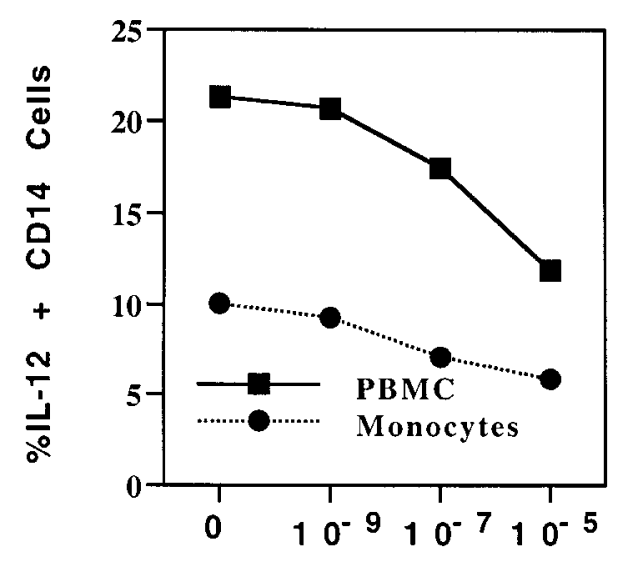

B

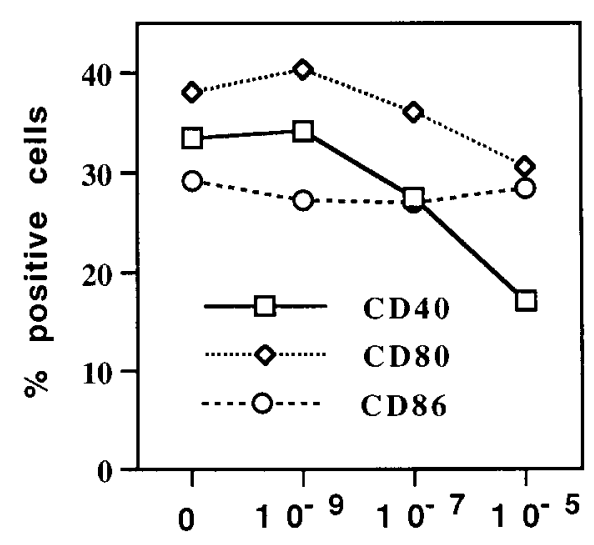

4 HC concentration (M)

Figure 5. Effect of 4HC on IL-12 and costimulatory molecules expression. (A) PBMCs (filled squares; $2 \times$ $10^{6} / \mathrm{ml}$ ) or purified CD $14^{+}$(filled circles; $2 \times 10^{6} / \mathrm{ml}$ ) were cultured for $1 \mathrm{~h}$ in the absence or presence of different concentrations of $4 \mathrm{HC}$ ( $\mathrm{x}$-axis). Cells were washed three times in complete culture media and then primed with rhIFN- $\gamma$ for $2 \mathrm{~h}$ and stimulated with LPS for $22 \mathrm{~h}$. (B) Purified monocytes were cultured for $1 \mathrm{~h}$ with various concentrations of $4 \mathrm{HC}$ (x-axis) and washed three times then incubated in culture media for $18 \mathrm{~h}$. Surface staining with CD40 (squares), CD80 (diamonds), and CD86 (circles) was performed as described in Methods. These results are representative of four independent experiments. 
The effect of $4 \mathrm{HC}$ on decreased IL-12 production may be secondary to decreased expression of costimulatory molecules. Thus, we examined the expression of CD40, CD80, CD86, and Class II MHC on monocytes after incubation with $4 \mathrm{HC}$ in vitro. As seen in Fig. $5 \mathrm{~B}$, incubation with $4 \mathrm{HC}$ induced a significant dose-dependent downregulation of CD40 expression on monocytes. A slight decrease in CD80 expression was seen at high doses. No change in CD86 or Class II MHC (not shown) expression was noted.

IL-12 expression in MS monocytes correlates with disease activity. We were interested in determining if there was a relationship between IL-12 production and disease activity. Patients were classified into two groups: active or stable by their treating physician at the time of blood drawing, and IL-12 expression was determined without knowledge of the clinical classification. IL-12 expression was compared among patients (treated or untreated) with stable disease $(n=36)$ and patients with active disease $(n=25)$. We found a statistically significant increase in IL-12 production in the group with active disease compared with patients with stable disease $(26.0 \pm 2.7$ vs. $18.3 \pm 1.8, P=0.02$; Fig. 6 ). There was no correlation between IL-12 production and EDSS score.

\section{Discussion}

In this study, we investigated cytokine production in PBMC from MS patients by using intracellular detection of cytokines by flow cytometry $(30,31)$. This method enables detection of cytokine production by individual cell populations and simultaneously determines their phenotype based upon surface antigen expression. Unstimulated monocytes produce little or no IL-12 spontaneously; thus we used in vitro stimulation to detect IL-12-secreting cells. NK cells did not produce IL-12 before or after stimulation, and a very small percentage of $\mathrm{B}$ cells $(<1 \%)$ had detectable IL-12 production.

We found that the frequency of IL-12-producing monocytes in untreated MS patients was higher than in controls.

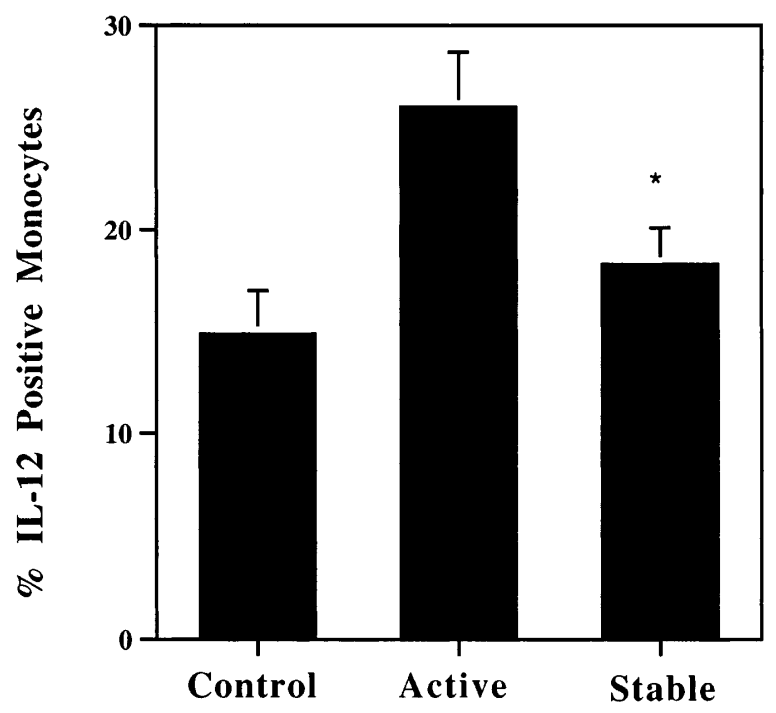

Figure 6. IL-12 expression correlates with disease activity. IL-12 production in monocytes of healthy individuals (Control), MS patients with active disease (Active) and stable disease (Stable); ${ }^{*} P=0.02$ for comparison between active and stable.
These results are in agreement with previous reports in which an elevated production of IL-12 by PBMC was found in progressive MS (16, 17). Furthermore, IL-12 expression segregated with the disease activity as assessed by the treating physician suggesting a role for IL-12 in disease progression. The frequency of TNF- $\alpha$-producing monocytes was not statistically different among the patient subgroups but showed a trend towards increased frequency in untreated progressive patients. This may be due to a selective effect of CY/MP on IL-12 production, to an effect of CY/MP on subpopulations of monocytes that produce mainly IL-12, or due to the smaller sample size analyzed for TNF- $\alpha$. We also found increased IFN- $\gamma-$ expressing T cells in patients with progressive MS on no treatment in agreement with a previous report of increased IFN- $\gamma$ mRNA-expressing PBMC in MS patients (32) and with the known deleterious effect of IFN- $\gamma$ in MS (33-35).

The second major finding in our study is the change in cytokine balance induced by immunosuppressive treatments. Treatment with methotrexate increased TGF- $\beta$ production by T cells. Methylprednisolone (MP) monthly treatment was associated with a decrease in IFN- $\gamma$-expressing cells and an increase in IL-4-expressing cells. Although in vitro decrease in IL-12 production by dexamethasone in monocytes has recently been reported (36), we did not observe any effect on IL-12 production in the group of patients treated with monthly MP. That may be explained by either a difference in the biological effects of MP and dexamethasone or a predominant effect of dexamethasone in the non-CD14 population of adherent cells. CY/MP treatment was associated with multiple cytokine effects. There was a significant decrease in IL-12 and IFN- $\gamma$ and an increase in IL-4, IL-5, and TGF- $\beta$. The latter was increased both in T cells and monocytes. Thus, CY/MP treatment was associated with a shift in cytokines from Th1 to Th2/Th3 pattern. These results agree with our recent report of increased antiCD3-induced IL-4 secretion by T cells in patients treated with cyclophosphamide (27). The increase in percentage of IL-4, IL-5, and TGF- $\beta$-producing cells in CY/MP treated patients was much less than the changes we observed for IL-12 and IFN- $\gamma$. Given the low numbers of IL-4-, IL-5-, and TGF- $\beta-$ secreting cells in untreated MS patients, the increases we observed are likely to be biologically significant, although the results should be interpreted with caution. The differences noted between untreated and CY/MP treated patients in percentage of cells producing IL-4- and IL-5-producing cells were seen under unstimulated conditions, no differences in stimulated IL-4- or IL-5-producing cells were noted. A similar phenomenon was reported in patients treated with $\operatorname{rIFN}-\beta_{1 \mathrm{~b}}$, where the percentage of monocytes spontaneously producing IL-10 was increased compared with untreated patients, but this difference disappeared after stimulation (37).

The finding of decreased IL-12 in CY/MP-treated patients also was observed in patients followed-up prospectively before and after treatment. In these patients the cytokine shifts occur subsequent to the start of treatment. Corticosteroids are known to inhibit the production of most cytokines in activated T cells $(38,39)$, and may increase IL-4 synthesis in T cells $(40$, 41). Because pulse cyclophosphamide treatment includes methylprednisolone infusions, we evaluated the effects of monthly MP treatment. We found that these patients had a modest but significant increase in IL-4 expression and a decrease in IFN- $\gamma$ expression. These results suggest that corticosteroids promote a Th2 cytokine response in $\mathrm{T}$ cells. Thus, 
methylprednisolone may have a synergistic effect with cyclophosphamide in inducing immune deviation.

TGF- $\beta$ is a regulator of immune responses and plays an important role in recovery from $\operatorname{EAE}(7,10,42,43)$, and investigators have reported that there is less severe disease in MS patients with increased levels of TGF- $\beta$ secreting cells (44). It was recently shown that IL-12 and IFN- $\gamma$ are important regulators of TGF- $\beta$ production both in vivo and in vitro (45). Thus, our finding of an increase in TGF- $\beta$ expression by T cells and monocytes in patients treated with CY/MP is consistent with the decreased IL-12 and IFN- $\gamma$ expression in these patients. Whether this increased production of TGF- $\beta$ is a direct effect of treatment or is secondary to the change in $\mathrm{Th} 1 / \mathrm{Th} 2$ balance remains to be determined.

In vitro studies suggest that the decreased IL-12 production is a direct effect of cyclophosphamide metabolite on monocytes. Thus addition of $4 \mathrm{HC}$ in vitro resulted in decreased IL-12 expression. $4 \mathrm{HC}$ is a synthetic compound that spontaneously hydrolyzes in aqueous solution to 4-hydroxycyclophosphamide, the initial metabolite formed by liver microsomal activation of cyclophosphamide. 4HC mimics the immunosuppressive effects of cyclophosphamide observed in the intact organism $(46,47)$. Surprisingly, the inhibition of IL-12 expression by $4 \mathrm{HC}$ in vitro was selective, because expression of other cytokines produced by monocytes, such as TNF- $\alpha$ and IL-10, was not affected. However, an indirect effect of cyclophosphamide treatment on other cytokine production cannot be ruled out. Purified stimulated monocytes had lower IL-12 expression compared with stimulation of PBMCs, probably reflecting the absence of signaling from CD40 ligand usually provided by $\mathrm{T}$ cells in the PBMC cultures. CD40-CD40L interaction is critical for IL-12 production by monocytes under antigen driven conditions $(16,48)$, but monocytes can produce IL-12 via a CD40/CD40L independent pathway in response to LPS or heat-killed Listeria monocytogenes, and this pathway does not require interaction of APC with $T$ cells (49). Nevertheless, IL-12 expression by purified monocytes was slightly decreased by the addition of the metabolite, suggesting some direct effect of cyclophosphamide on monocytes. Although the mechanism by which cyclophosphamide affects IL-12 secretion remains to be defined, it is unlikely to be due to the alkylating properties of cyclophosphamide, as proliferating cells are more vulnerable to the cytotoxic effects of cyclophosphamide, and monocytes are not considered to be actively proliferating cells. Furthermore, in vitro addition of $4 \mathrm{HC}$ to the cell cultures was not associated with decreased cell viability. Thus, cyclophosphamide may have a direct effect on monocyte IL-12 production and an indirect effect ( $\mathrm{T}$ cell dependent) through decreased CD40 expression on monocytes.

In summary, our results demonstrate a Th1-type cytokine bias in peripheral blood mononuclear cells of untreated progressive MS patients that is reversed by CY/MP treatment and is associated with Th2 and TGF- $\beta$ (Th3) type responses. These findings provide a basis for immune monitoring of patients with MS. If the linkage between IL-12 production and response to treatment is validated in longitudinal studies, this would suggest that treatments that downregulate IL-12 are likely to be beneficial in progressive MS.

\section{Acknowledgments}

Supported by National Institutes of Health (NS-23132-11A), the National Multiple Sclerosis Society, and the Nancy Davis Center With- out Walls. M.C. was supported by a grant from the Fondo de Investigacion Sanitaria (ref. 97/5462).

\section{References}

1. Khoury, S.J., H.L. Weiner, and D.A. Hafler. 1996. Immunological mechanisms in multiple sclerosis. In Handbook of Multiple Sclerosis. S.D. Cook, editor. Marcell Dekker Inc., New York. 145-155.

2. Mosmann, T.R., H. Cherwinski, M.W. Bond, M.A. Giedlin, and R.L. Coffman. 1986. Two types of murine helper T cell clone. I. Definition according to profiles of lymphokine activities and secreted proteins. J. Immunol. 136: 2348-2357.

3. Chen, Y., V.K. Kuchroo, J.-I. Inobe, D.A. Hafler, and H.L. Weiner. 1994. Regulatory T cell clones induced by oral tolerance: suppression of autoimmune encephalomyelitis. Science. 265:1237-1240.

4. Weiner, H.L. 1997. Oral tolerance: immune mechanisms and treatment of autoimmune diseases. Immunol. Today. 18:335-343.

5. Ando, D.G., J. Clayton, D. Kono, J.L. Urban, and E.E. Sercarz. 1989. Encephalitogenic T cells in the B10.PL model of experimental allergic encephalomyelitis (EAE) are of the Th-1 lymphokine subtype. Cell. Immunol. 124:132143.

6. Racke, M.K., A. Bonomo, D.E. Scott, B. Cannella, A. Levine, C.S Raine, E.M. Shevach, and M. Rocken. 1994. Cytokine-induced immune deviation as a therapy for inflammatory autoimmune disease. J. Exp. Med. 180:19611966.

7. Khoury, S.J., W.W. Hancock, and H.L. Weiner. 1992. Oral tolerance to myelin basic protein and natural recovery from experimental autoimmune encephalomyelitis are associated with downregulation of inflammatory cytokines and differential upregulation of transforming growth factor beta, interleukin 4, and prostaglandin E expression in the brain. J. Exp. Med. 176:1355-1364.

8. Kennedy, M.K., D.S. Torrance, K.S. Picha, and K.M. Mohler. 1992. Analysis of cytokine mRNA expression in the central nervous system of mice with experimental autoimmune encephalomyelitis reveals that IL-10 mRNA expression correlates with recovery. J. Immunol. 149:2496-2505.

9. Issazadeh, S., A. Ljungdahl, B. Hojeberg, M. Mustafa, and T. Olsson. 1995. Cytokine production in the central nervous system of Lewis rats with experimental autoimmune encephalomyelitis: dynamics of mRNA expression for interleukin 10 , interleukin 12 , cytolysin, tumor necrosis factor-a and tumor necrosis factor-b. J. Neuroimmunol. 61:205-212.

10. Chen, Y., W.W. Hancock, R. Marks, P.A. Gonnella, and H.L. Weiner 1998. Mechanisms of recovery from experimental allergic encephalomyelitis: T cell deletion and immune deviation in myelin basic protein receptor transgenic mice. J. Neuroimmunol. 82:149-159.

11. Manetti, R., P. Parronchi, M.G. Giudizi, M.P. Piccinni, E. Maggi, G. Trinchieri, and S. Romagnani. 1993. Natural killer cell stimulatory factor (interleukin 12 [IL-12]) induces T helper type 1 (Th1)-specific immune responses and inhibits the development of IL-4-producing Th cells. J. Exp. Med. 177:11991204.

12. Manetti, R., F. Gerosa, M.G. Giudizi, R. Biagiotti, P. Parronchi, M.P Piccinni, S. Sampognaro, E. Maggi, S. Romagnani, G. Trinchieri, et al. 1994. Interleukin 12 induces stable priming for interferon gamma (IFN-gamma) production during differentiation of human T helper (Th) cells and transient IFNgamma production in established Th2 cell clones. J. Exp. Med. 179:1273-1283.

13. Seder, R.A., R. Gazzinelli, A. Sher, and W.E. Paul. 1993. Interleukin 12 acts directly on $\mathrm{CD} 4+\mathrm{T}$ cells to enhance priming for interferon gamma production and diminishes interleukin 4 inhibition of such priming. Proc. Natl. Acad. Sci. USA. 90:10188-10192.

14. Hsieh, C.S., S.E. Macatonia, C.S. Tripp, S.F. Wolf, A. O'Garra, and K.M. Murphy. 1993. Development of TH1 CD4+ T cells through IL-12 produced by Listeria-induced macrophages. Science. 260:547-549.

15. Leonard, J.P., K.E. Waldburger, and S.J. Goldman. 1995. Prevention of experimental autoimmune encephalomyelitis by antibodies against interleukin 12. J. Exp. Med. 181:381-386.

16. Balashov, K.E., D.R. Smith, S.J. Khoury, D.A. Hafler, and H.L. Weiner. 1997. Increased interleukin 12 production in progressive multiple sclerosis: induction by activated CD4+ T cells via CD40 ligand. Proc. Natl. Acad. Sci. USA. 94:599-603.

17. Nicoletti, F., F. Patti, C. Cocuzza, P. Zaccone, A. Nicoletti, R. DiMarco, and A. Reggio. 1996. Elevated serum levels of interleukin-12 in chronic progressive multiple sclerosis. J. Neuroimmunol. 70:87-90.

18. Paterson, P.Y., and M.A. Hanson. 1969. Cyclophosphamide inhibition of experimental allergic encephalomyelitis and cellular transfer of the disease in Lewis rats. J. Immunol. 103:1311-1316.

19. Paterson, P.Y., and D.G. Drobish. 1969. Cyclophosphamide: effect on experimental allergic encephalomyelitis in Lewis rats. Science. 165:191-192.

20. Brinkman, C.J., W.M. Nillesen, and O.R. Hommes. 1984. The effect of cyclophosphamide on T lymphocytes and T lymphocyte subsets in patients with chronic progressive multiple sclerosis. Acta Neurol. Scand. 69:90-96.

21. Moody, D.J., J.L. Fahey, E. Grable, G.W. Ellison, and L.W. Myers. 1987. Administration of monthly pulses of cyclophosphamide in multiple sclerosis patients. Delayed recovery of several immune parameters following dis- 
continuation of long-term cyclophosphamide treatment. J. Neuroimmunol. 14: $175-182$.

22. Lamers, K.J., B.M. Uitdehaag, O.R. Hommes, W. Doesburg, R.A. Wevers, and W.J. von Geel. 1988. The short-term effect of an immunosuppressive treatment on CSF myelin basic protein in chronic progressive multiple sclerosis. J. Neurol. Neurosurg. Psychiatry. 51:1334-1337.

23. Hafler, D.A., J. Orav, R. Gertz, L. Stazzone, and H.L. Weiner. 1991. Immunologic effects of cyclophosphamide/ACTH in patients with chronic progressive multiple sclerosis. J. Neuroimmunol. 32:149-158.

24. Hauser, S.L., D.M. Dawson, J.R. Lehrich, M.F. Beal, S.V. Kevy, R.D. Propper, J.A. Mills, and H.L. Weiner. 1983. Intensive immunosuppression in progressive multiple sclerosis. A randomized, three-arm study of high-dose intravenous cyclophosphamide, plasma exchange, and ACTH. N. Engl. J. Med. 308:173-180.

25. Weiner, H.L. G.A. Mackin, E.J. Orav, D.A. Hafler, D.M. Dawson, Y. LaPierre, R. Herndon, J.R. Lehrich, S.L. Hauser, A. Turel, et al. 1993. Intermittent cyclophosphamide pulse therapy in progressive multiple sclerosis: final report of the Northeast Cooperative Multiple Sclerosis Treatment Group. Neurology. 43:910-918.

26. Group., T.C.C.M.S.S. 1991. The Canadian Cooperative trial of cyclophosphamide and plasma exchange in progressive multiple sclerosis. Lancet. 337:442-446.

27. Smith, D.R., K.E. Balashov, D.A. Hafler, S.J. Khoury, and H.L. Weiner. 1997. Immune deviation following pulse cyclophosphamide/methylprednisolone treatment of multiple sclerosis: increased interleukin-4 production and associated eosinophilia. Ann. Neurol. 42:313-318.

28. Takashima, H., D. Smith, H. Fukaura, S. Khoury, D. Hafler, and H. Weiner. 1998. Pulse cyclophosphamide plus methylprednisolone induces myelin antigen specific IL-4 secreting T cells in multiple sclerosis patients. Clin. Immunol. Immunopathol. 88:28-34.

29. Lewis, C.E. 1991. Detecting cytokine production at the single-cell level. Cytokine. 3:184-188

30. Prussin, C. 1997. Cytokine flow cytometry: understanding cytokine biology at the single-cell level. J. Clin. Immunol. 17:195-204.

31. Jung, T., U. Schauer, C. Heusser, C. Neumann, and C. Rieger. 1993. Detection of intracellular cytokines by flow cytometry. J. Immunol. Meth. 159:197207.

32. Link, J., M. Soderstrom, T. Olsson, B. Hojeberg, A. Ljungdahl, and H. Link. 1994. Increased transforming growth factor-beta, interleukin-4, and interferon-gamma in multiple sclerosis. Ann. Neurol. 36:379-386.

33. Panitch, H.S., R.L. Hirsch, A.S. Haley, and K.P. Johnson. 1987. Exacerbations of multiple sclerosis in patients treated with gamma interferon. Lancet. $1: 893-895$

34. Beck, J., P. Rondot, L. Catinot, E. Falcoff, H. Kirchner, and J. Wietzerbin. 1988. Increased production of interferon gamma and tumor necrosis factor precedes clinical manifestations in multiple sclerosis: do cytokines trigger off exacerbations? Acta Neurol. Scand. 78:318-323.

35. Olsson, T., W.-Z. Wang, B. Höjeberg, V. Kostulas, Y.-P. Jiang, G. Anderson, H.-P. Ekre, and H. Link. 1990. Autoreactive T-lymphocytes in multiple sclerosis determined by antigen-induced secretion of interferon-gamma. $J$. Clin. Invest. 86:981-985.
36. Blotta, M.H., R.H. DeKruyff, and D.T. Umetsu. 1997. Corticosteroids inhibit IL-12 production in human monocytes and enhance their capacity to induce IL-4 synthesis in CD4+ lymphocytes. J. Immunol. 158:5589-5595.

37. Crucian, B., P. Dunne, H. Friedman, R. Ragsdale, S. Pross, and R. Widen. 1996. Detection of altered T helper 1 and $\mathrm{T}$ helper 2 cytokine production by peripheral blood mononuclear cells in patients with multiple sclerosis utilizing intracellular cytokine detection by flow cytometry and surface marker analysis. Clin. Diagn. Lab. Immunol. 3:411-416.

38. Scheinman, R.I., P.C. Cogswell, A.K. Lofquist, and A.J. Baldwin. 1995. Role of transcriptional activation of I kappa B alpha in mediation of immunosuppression by glucocorticoids. Science. 270:283-286.

39. Auphan, N., J.A. DiDonato, C. Rosette, A. Helmberg, and M. Karin 1995. Immunosuppression by glucocorticoids: inhibition of NF-kappa B activity through induction of I kappa B synthesis. Science. 270:286-290.

40. Ramirez, F., D.J. Fowell, M. Puklavec, S. Simmonds, and D. Mason. 1996. Glucocorticoids promote a TH2 cytokine response by CD4+ T cells in vitro. J. Immunol. 156:2406-2412.

41. Zieg, G., G. Lack, R.J. Harbeck, E.W. Gelfand, and D.Y. Leung. 1994 In vivo effects of glucocorticoids on IgE production. J. Allergy Clin. Immunol. 94:222-230

42. Racke, M.K. S. Dhib-Jalbut, P.S. Cannella, P.S. Albert, and D.E. McFarlin. 1991. Prevention and treatment of chronic relapsing experimental allergic encephalomyelitis by transforming growth factor- $\beta 1$. J. Immunol. 146:30123017.

43. Johns, L., K. Flanders, G. Ranges, and S. Sriram. 1991. Successful treatment of experimental allergic encephalomyelitis with transforming growth fac tor- $\beta 1$. J. Immunol. 147:1792-1796.

44. Olsson, T. 1995. Critical influences of the cytokine orchestration on the outcome of myelin antigen-specific T-cell autoimmunity in experimental autoimmune encephalomyelitis and multiple sclerosis. Immunol. Rev. 144:245268.

45. Marth, T., W. Strober, R.A. Seder, and B.L. Kelsall. 1997. Regulation of transforming growth factor-beta production by interleukin-12. Eur. J. Immunol. 27:1213-1220.

46. Diamantstein, T., M. Klos, H. Hahn, and S.H. Kaufmann. 1981. Direct in vitro evidence for different susceptibilities to 4-hydroperoxycyclophosphamide of antigen-primed $\mathrm{T}$ cells regulating humoral and cell-mediated immune responses to sheep erythrocytes: a possible explanation for the inverse action of cyclophosphamide on humoral and cell-mediated immune responses. J. Immunol. 126:1717-1719.

47. Kaufmann, S.H., H. Hahn, and T. Diamantstein. 1980. Relative susceptibilities of $\mathrm{T}$ cell subsets involved in delayed-type hypersensitivity to sheep red blood cells to the in vitro action of 4-hydroperoxycyclophosphamide. J. Immunol. 125:1104-1108.

48. Cella, M., D. Scheidegger, L.K. Palmer, P. Lane, A. Lanzavecchia, and G. Alber. 1996. Ligation of CD40 on dendritic cells triggers production of high levels of interleukin-12 and enhances T cell stimulatory capacity: T-T help via APC activation. J. Exp. Med. 184:747-752.

49. DeKruyff, R.H., R.S. Gieni, and D.T. Umetsu. 1997. Antigen-driven but not lipopolysaccharide-driven IL-12 production in macrophages requires triggering of CD40. J. Immunol. 158:359-366. 